\title{
High-frequency sonography in the evaluation of nail psoriasis
}

\author{
Mihaela Elena Marina*1, Carolina Solomon², Sorana-Daniela Bolboaca*3, Corina Bocsa ${ }^{4}$, \\ Carmen Mihaela Mihu', Alexandru Dumitru Tătaru ${ }^{5}$
}

\author{
${ }^{1}$ Histology Department, ${ }^{2}$ Radiology Department, ${ }^{3}$ Medical Informatics and Biostatistics Department, ${ }^{5}$ Dermatology \\ Department, "Iuliu Haţieganu" University of Medicine and Pharmacy, ${ }^{4}$ Medical-Surgical Center InterServisan, \\ Cluj-Napoca, Romania \\ * the authors shared the first authorship
}

\begin{abstract}
Aim: To evaluate the morphostructural aspects and nail vascularity in the nail unit of patients with psoriasis, and to evaluate whether there are differences among psoriatic patients with and without nail involvement. Material and methods: Nail plates and nail bed changes, nailfold vessel resistance index (NVRI), power and color Doppler blood flow appearances were investigated in 23 patients with moderate-to-severe psoriasis, with and without nail involvement, and compared to those of 11 healthy participants. Results: Ventral nail plate deposits were present only in psoriasis patients. Irregular or totally fused nail plates and increased nail plate thickness was frequently observed in psoriasis patients compared to controls. NVRI was increased in psoriatic patients' nails compared to controls $(0.62 \mathrm{vs} .0 .57, \mathrm{p}<0.0001)$. In the psoriasis patient group there was significant statistical difference in NVRI in patients with nail involvement compared to those without ( 0.66 vs. 0.55 , $\mathrm{p}<0.0001)$. Conclusions: High-frequency gray scale sonography provides valuable information regarding morphostructural changes in nail unit structure in patients with psoriasis. Power Doppler imaging enables blood flow assessment in psoriasis nail induced changes.
\end{abstract}

Keywords: nailfold vessel resistance index (NVRI), nail psoriasis, power and color Doppler sonography

\section{Introduction}

Psoriasis is a chronic, relapsing skin disease affecting millions of people worldwide, with a general prevalence estimated to be approximately $2-3 \%$ [1]. While skin lesions are the most typical findings of psoriasis, nail involvement is an often-overlooked clinical manifestation of the disease. Nail changes were reported to occur in up to $40 \%$ of patients with mild psoriasis and $50-70 \%$ with severe disease $[2,3]$. Samman et al reported an $80 \%$ to $90 \%$ lifetime incidence of nail involvement in psoriatic

Received 09.03.2016 Accepted 30.04.2016

Med Ultrason

2016, Vol. 18, No 3, 312-317

Corresponding author: Carolina Solomon

Department of Radiology, "Iuliu Haţieganu"

University of Medicine and Pharmacy

3-5 Clinicilor Street

400006 Cluj-Napoca, Romania

Phone: +40745601976

E-mail: carolina.solomon@umfcluj.ro patients [4]. Nail psoriasis, in the absence of cutaneous lesions, can be present in 5\% of patients [5] and represents a special diagnostic challenge.

Traditionally, nail psoriasis is a clinical diagnosis due to the fact that nail biopsy, besides being a bleeding and painful procedure, may cause detrimental cosmetic changes such as scarring and permanent nail dystrophy [6]. Dermoscopy and videodermoscopy, generally used in describing benign and malignant tumoral skin lesions, were reported as being non-invasive, quickly applied, and easy-to-use methods that may aid in diagnosing nail psoriasis [7-10]. Capillaroscopy, usually used to study the microcirculation in collagen disease, can also be used to detect psoriasis induced microvascular changes, such as decreased capillary density and avascular areas in the periungual area [11].

High-resolution ultrasonography (HRUS) was used in assessing nail apparatus anatomy and soft tissues changes in patients with different nail pathologies and provided appropriate diagnosis and additional informa- 
tion to the clinical examination [12]. Easy to perform and having no risk of irradiation, HRUS has been proposed as a valuable imaging method in assessing the extension and activity of both nail and cutaneous psoriasis $[13,14]$.

The aim of this study was to evaluate both the morphological appearance and blood flow changes in the nail apparatus of patients with psoriasis compared to disease free controls using gray-scale and color and power Doppler HRUS.

\section{Material and methods}

\section{Study design}

This was a single center, transversal, observational study, from July 2014 until March 2015. Twenty-three adult patients (age $>18$ years old), with moderate-to-severe chronic plaque psoriasis measured by Psoriasis Area and Severity Index (PASI $>10)$, lasting at least 6 months, with or without fingernail involvement were included in the case group. Patients with clinical psoriatic arthritis or other inflammatory conditions affecting joints, such as rheumatoid arthritis, were excluded. Patients with diabetes, arterial hypertension, smokers (patients who smoked cigarettes daily or who had stopped smoking $<10$ years before enrollment in the study), and patients following systemic or topical treatment on their nails (for nail psoriasis) at the time of the clinical examination or in the last 3 month prior to examination, or having a skin disease that is associated with nail involvement and those who used artificial nails in the past 6 months were also excluded. The control group consisted of eleven nonsmoking healthy subjects, age and sex matched with the cases, without psoriatic arthritis, diabetes, or arterial hypertension. The University Ethics Committee approved the study and all subjects gave their written consent prior to the enrollment.

\section{Clinical evaluation}

All subjects were interviewed to collect demographical data: age, gender, age of onset and duration of psoriasis, age of onset, and duration of fingernail psoriasis. The severity of skin psoriasis was assessed using PASI (ranging from 0- no disease to 72- maximal disease), a score that combines the severity (erythema, induration, and desquamation) and percentage of affected area [15]. Onycomycosis was excluded by direct microscopic examination $(10 \%$ potassium hydroxide) in patients with nail bed hyperkeratosis.

\section{Ultrasonography assessment}

Real-time HRUS was performed by an experienced sonographer with more than 15 years' experience using an Ultrasonix Sonotouch scanner (Ultrasonix Medical Corporation, Richmond, Canada) equipped with a varia- ble-frequency transducer ranging from 8 to $40 \mathrm{MHz}$ (focal range $0.2-3 \mathrm{~cm}$, image field $16 \mathrm{~mm}$ ) to visualize nail anatomy.

In order to observe the blood flow a Hitachi EUB 8500 System equipped with a variable-frequency transducer ranging from $6.5-13 \mathrm{MHz}$ was used. The imaging parameters for Doppler ultrasound examinations were set to increase the detection of low-velocity, low-volume flows within the small inside of the nail bed (color Doppler: PRF 500-1000 Hz, wall filter 25-50 Hz, power Doppler: PRF $350-700 \mathrm{~Hz}$, wall filter $22-50 \mathrm{~Hz}$; color and power Doppler: color gain maximized for optimal sensitivity while avoiding excessive color noise, color vs. echo priority ranging from 70 to $90 \%$ and color persistence adjusted to high values).

The sonographic examinations were performed in a room with a constant controlled temperature of $24^{\circ} \mathrm{C}$, after a 20 minute rest period. The patient was seated, with the forearm in a neutral position over the table and the nails were scanned on longitudinal and transverse planes. The ultrasound gel had sufficient quantity so that the transducer exerted no compression, to avoid alteration of nail thickness or blood flow.

Each fingernail was scanned in the gray scale mode with $40 \mathrm{MHz}$ frequency transducer to detect morphostructural changes (deposits in dorsal nail plate, nail plates irregularities and thickness, nail bed thickness), and afterwards with the power Doppler technique to enable blood flow visualization. Nail plate thickness represents the distance between the two nail plates. Nail bed thickness was obtained by measuring the distance from the ventral nail plate to the dorsal of the distal phalanx at $2.5 \mathrm{~mm}$ from the proximal nail fold.

Using Doppler examination, in the proximal third of the nail plate of each fingernail, the maximum speeds of flow in systole $(\mathrm{Qs})$ and in diastole $(\mathrm{Qd})$ were registered and the nailfold vessel resistance index (NVRI) was assessed as the following ratio: (Qs-Qd)Qs. The NVRI ratio ranges from 0 (no resistance to blood flow at all: no changes in the circulation of blood in systole and diastole) to 1 (maximum resistance to blood flow circulation of blood only at systole) [16]. Color and Power Doppler spots in nail bed were registered as follows: low $<25 \%$, medium $25-50 \%$, and multiple $>50 \%$ of nail bed's area occupied by vascular structures.

\section{Statistical analysis}

Descriptive and inferential statistical methods were applied using Statistica software (version 8, StatSoft, USA). Qualitative data were summarized as percentages and associated 95\% confidence interval (provided in squared brackets along the manuscript) computed with an exact method [17]. The normal distribution of 
quantitative data was tested using the Shapiro-Wilks test and whenever the p-value was higher than $5 \%$, data were considered normally distributed. Quantitative data that proved normally distributed were summarized as mean \pm standard deviation; otherwise median and interquartile range (expressed as Q1-Q3, where Q1 is the first quartile and Q3 is the third quartile) was used. Comparisons between groups were conducted with $\mathrm{Z}$ test for proportions (qualitative data), student t-test for independent samples for quantitative normally distributed data, and respectively Mann-Whitney test for quantitative data when Shapiro-Wilks null hypothesis (data follow normal distribution) failed to be rejected. Inferential statistical analysis was conducted at a significance level of 5\%, and any $\mathrm{p}$-value lower than 0.05 was considered statistically significant.

\section{Results}

Twenty-three patients with cutaneous psoriasis (14 with nail psoriasis and 9 without nail involvement) and eleven healthy subjects were included in the study. Thus, on the sample there were evaluated 122 nails belonging

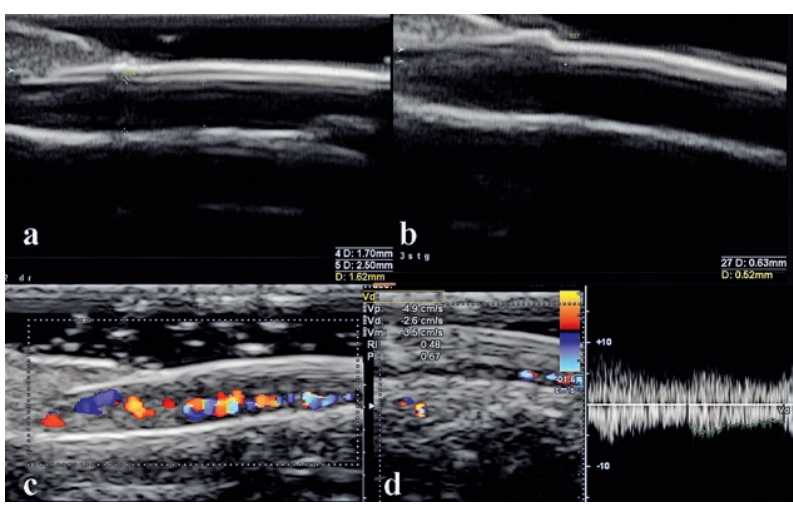

Fig 1. Normal US nail aspect in a non affected 47 years old woman: a) 2D US - thickness of the nail bed; b) 2D US - measurement of the nail plaques thickness; c) color Doppler US aspect of the vessels in the nail bed; d) spectral Doppler - measurement of the Doppler indices in nail vessels to patients with psoriasis and 82 nails belonging to controls.

Mean age in the control group was 46.09 years ( $\pm 11.82 \mathrm{SD}$ ), ranging from 20 to 59 years old, without significant differences when compared with patients in the psoriasis group $(52.43 \pm 14.28 \mathrm{SD})$.

Demographic and disease characteristics of the patients in psoriasis group are summarized in Table I.

No statistical difference was observed between genders in the psoriasis patients, regarding mean age of onset of cutaneous psoriasis, mean duration of psoriasis or PASI score.

In the control group there were no ventral nail plate deposits or irregularities in any of the nail plates $(\mathrm{p}<0.05)$, and only a low number of spots on Color and Power Doppler imaging (fig 1) were detected.

In the psoriasis group $13.11 \%$ of the investigated nails presented deposits in the ventral plate; both of the nail plates had various degrees of involvement and the nail plates were significantly thicker compared to controls. Color and power Doppler assessment revealed an increased nail blood flow in psoriasis patients with a higher vascular resistance when compared to controls (fig 2, Table II).

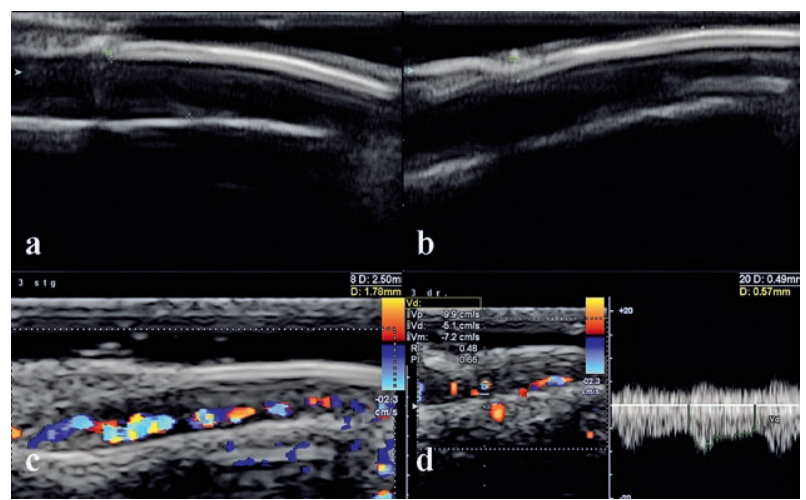

Fig 2. US aspect in a 52 years old female with psoriasis and no clinical affected nails: a) 2D US - thick-ness of the nail bed; b) 2D US - measurements of the nail plaques thickness; c) color Doppler US - tortuous vessels in the nail bed; d) spectral Doppler - Doppler indices in nail vessels

Table I. Characteristics of patients in the psoriasis group

\begin{tabular}{lllll}
\hline & $\begin{array}{l}\text { All } \\
(\mathbf{n = 2 3 )}\end{array}$ & $\begin{array}{l}\text { With nail involvement } \\
(\mathbf{n = 1 4 )}\end{array}$ & $\begin{array}{l}\text { Without nail involvement } \\
(\mathbf{n = 9})\end{array}$ & p-value \\
\hline Age (years) & $52.43 \pm 14.28$ & $52.57 \pm 17.96$ & $52.22 \pm 6.04$ & $0.056(0.95)$ \\
Gender & & & & $|1.72|(0.08)$ \\
$\quad$ F & 35 & 21 & 56 & \\
M & 65 & 79 & 44 & $-2.21(0.03)$ \\
Mean age of onset of cutaneous psoriasis & $36.39 \pm 12.69$ & $32.07 \pm 12.68$ & $43.11 \pm 9.91$ & $2.17(0.04)$ \\
Mean duration of cutaneous psoriasis & $15.96 \pm 13.10$ & $20.36 \pm 14.47$ & $9.11 \pm 6.70$ & $1.67(0.10)$ \\
PASI & $17.81 \pm 3.64$ & $18.79 \pm 3.37$ & $16.29 \pm 3.70$ & \\
\hline
\end{tabular}

Data are expressed as mean \pm standard deviation; a: \%, Z-test for proportions; PASI = Psoriasis Area and Severity Index, F - female, M - male 
Table II. Nail US morphostructural and blood flow characteristics: cases versus controls

\begin{tabular}{|c|c|c|c|}
\hline & Psoriasis $(n=122)$ & Control $(n=82)$ & Stat (p-value) \\
\hline Ventral nail plate deposits ${ }^{\mathrm{a}}$ & $13.11[7.38-20.49]$ & $0[0.00-4.86]$ & $3.41(0.0006)$ \\
\hline \multicolumn{4}{|l|}{ Nail plates aspect ${ }^{\mathrm{a}}$} \\
\hline 1 (regular) & $24.59[17.22-32.78]$ & $100.00[95.14-100]$ & $-10.61(<0.0001)$ \\
\hline 2 (irregular) & $34.43[26.24-43.44]$ & $0.00[0.00-4.86]$ & $5.96(<0.0001)$ \\
\hline 3 (pointly fused) & $34.43[26.24-43.44]$ & $0.00[0.00-4.86]$ & $5.96(<0.0001)$ \\
\hline 4 (totally fused) & $6.56[2.47-12.29]$ & $0.00[0.00-4.86]$ & $2.36(0.0182)$ \\
\hline \multicolumn{4}{|l|}{ CD spots ${ }^{\mathrm{a}}$} \\
\hline 1 (low) & $36.07[27.88-45.08]$ & $100.00[95.14-100]$ & $-9.21(<0.0001)$ \\
\hline 2 (medium) & $54.92[45.91-63.93]$ & $0.00[0.00-4.86]$ & $8.19(<0.0001)$ \\
\hline 3 (multiple) & $9.02[4.11-15.57]$ & $0.00[0.00-4.86]$ & $2.79(0.0052)$ \\
\hline \multicolumn{4}{|l|}{ PD spots ${ }^{\mathrm{a}}$} \\
\hline 1 (low) & $36.89[28.70-45.90]$ & $100.00[95.14-100]$ & $-9.12(<0.0001)$ \\
\hline 2 (medium) & $54.10[45.09-63.11]$ & $0.00[0.00-4.86]$ & $8.10(<0.0001)$ \\
\hline 3 (multiple) & $9.02[4.11-15.57]$ & $0.00[0.00-4.86]$ & $2.79(0.0052)$ \\
\hline Nail bed thickness ${ }^{b}$ & $1.88(1.71-2.03)$ & $1.89(1.78-2.00)$ & $-0.74(0.4621)$ \\
\hline Nail plates thickness ${ }^{b}$ & $0.86(0.60-1.14)$ & $0.63(0.59-0.67)$ & $5.68(<0.0001)$ \\
\hline $\mathrm{NVRI}^{\mathrm{b}}$ & $0.62(0.55-0.69)$ & $0.57(0.55-0.58)$ & $4.75(<0.0001)$ \\
\hline
\end{tabular}

$\mathrm{CD}=$ Color Doppler; PD = Power Doppler; NVRI = nailfold vessel resistance index; a: \% [95\%CI], where CI = confidence interval; comparisons done with Z-test; b: median (Q1-Q3), where Q1 = first statistic, Q3 = third statistic; Mann-Whitney test for comparison between psoriasis and control group

Table III. Nail US morphostructural and blood flow characteristics in patients with psoriasis, with and without nail involvement

\begin{tabular}{|c|c|c|c|}
\hline & $\begin{array}{l}\text { Nail involvement } \\
(n=79)\end{array}$ & $\begin{array}{l}\text { Without nail involvement } \\
(n=43)\end{array}$ & Statistic (p-value) \\
\hline Ventral nail plate deposits ${ }^{\mathrm{a}}$ & $17.72[10.14-27.83]$ & $4.65[0.05-16.23]$ & $2.04(0.0410)$ \\
\hline \multicolumn{4}{|l|}{ Nail plates aspect ${ }^{\mathrm{a}}$} \\
\hline 1 (regular) & $26.58[17.74-37.96]$ & $20.93[9.36-34.83]$ & $0.69(0.4887)$ \\
\hline 2 (irregular) & $36.71[26.60-48.09]$ & $30.23[16.33-46.46]$ & $0.72(0.4697)$ \\
\hline 3 (pointly fused) & $29.11[19.00-40.49]$ & $44.19[27.96-60.41]$ & $-1.67(0.094)$ \\
\hline 4 (totally fused) & $7.59[2.55-15.17]$ & $4.65[0.05-16.23]$ & $0.63(0.5307)$ \\
\hline \multicolumn{4}{|l|}{ CD spots ${ }^{\mathrm{a}}$} \\
\hline 1 (low) & $34.18[24.07-45.55]$ & $39.53[25.64-55.76]$ & $-0.59(0.5566)$ \\
\hline 2 (medium) & $55.70[44.32-67.07]$ & $53.49[37.26-69.71]$ & $0.23(0.8147)$ \\
\hline 3 (multiple) & $10.13[3.81-18.97]$ & $6.98[2.38-18.55]$ & $0.58(0.5618)$ \\
\hline \multicolumn{4}{|l|}{ PD spots ${ }^{\mathrm{a}}$} \\
\hline 1 (low) & $34.18[24.07-45.55]$ & 41.86 [27.96-58.09] & $-0.84(0.4010)$ \\
\hline 2 (medium) & $55.70[44.32-67.07]$ & $51.16[34.94-67.39]$ & $0.48(0.6307)$ \\
\hline 3 (multiple) & $10.13[3.81-18.97]$ & $6.98[2.38-18.55]$ & $0.58(0.5618)$ \\
\hline Nail bed thickness ${ }^{b}$ & $1.82(1.66-2.00)$ & $1.94(1.78-2.06)$ & $-1.82(0.0684)$ \\
\hline Nail plates thickness ${ }^{\mathrm{b}}$ & $0.93(0.69-1.24)$ & $0.71(0.51-0.97)$ & $3.38(0.0007)$ \\
\hline NVRI $^{b}$ & $0.66(0.60-0.71)$ & $0.55(0.48-0.60)$ & $4.68(<0.0001)$ \\
\hline
\end{tabular}

$\mathrm{CD}=$ Color Doppler; PD = Power Doppler; NVRI = nailfold vessel resistance index; a: \% [95\%CI], where CI = confidence interval; comparisons done with Z-test; b: median (Q1-Q3), where Q1 = first quartile, Q3 = third quartile; Mann-Whitney test for comparison between psoriasis and control group.

Among patients with psoriasis, those who have had clinical fingernail involvement presented obvious morphostructural US modifications: ventral nail plate deposits, irregular or totally fused together nail plates were frequently observed in those with nail involvement compared to those without nail involvement.

Vascularization's assessment revealed significant differences between the two subgroups in psoriatic patients: those with clinical nail involvement displayed a great number of medium and multiple CD and PD spots, while those without nail involvement displayed more often a low number of spots on CD and PD imaging (fig 3).

The vascular resistance to blood flow measured by NVRI proved to be significantly higher in patients with psoriasis compared to controls, and among patients with psoriasis: those with nail involvement showed a higher mean \pm SD than those without (Table III). 


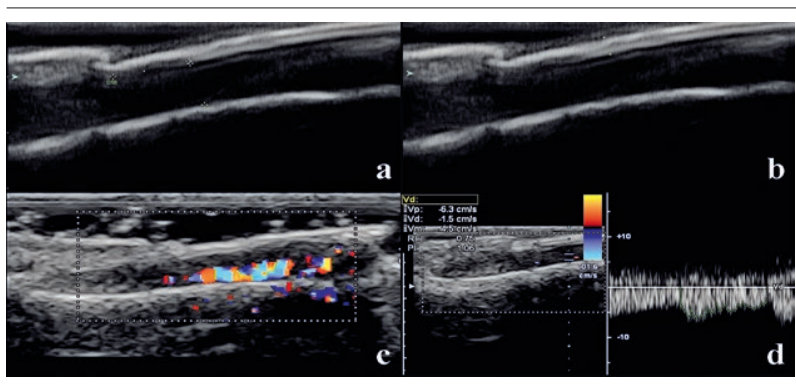

Fig 3. US aspect in a 59 years old male with psoriasis and with clinical affected nails: a) 2D US - thick-ness of the nail bed; b) 2D US - measurements of the nail plaques thickness; c) color Doppler US - tortuous vessels in the nail bed; d) spectral Doppler - Doppler indices in nail vessels

\section{Discussions}

Due to its major negative impact on the physical and social quality of life, in the last decade, there has been an increasing interest in assessing nail involvement in psoriatic patients. High-frequency ultrasonography probes and sensitive color and power Doppler technique permitted us to quantify both morphostructural and blood flow changes in the nail unit in patients with chronic moderate-to-severe cutaneous psoriasis.

Gray scale sonographic evaluation in nail apparatus showed deposits in the ventral nail plate only in patients with psoriasis, while none of the controls presented these changes. Moreover, among patients with psoriasis, the presence of ventral nail plate deposits were registered in $17.72 \%$ of the involved nails and in $4.62 \%$ of the nails without clinical signs of involvement $(p<0.05)$. These changes suggest the fact that HRUS can detect subclinical modifications in ventral nail plate in patients with cutaneous psoriasis but without obvious clinical involvement.

We found increased nail plate thickness in patients with psoriasis compared to healthy controls, with a significant difference between those with and without clinical nail involvement, similar data being also reported by Gisondi et al [18]. In contrast with the same study, we did not find increased nail bed thickness in patients with psoriasis, even though this modification was expected. This fact could be explained by different anatomical sites of ultrasound measurement (we performed this measurement at $2.5 \mathrm{~mm}$ from the proximal nail fold) or by different stages of the nail disease (active or passive phase onichopathy). Also, the small number of enrolled patients and controls in our study could have influenced this outcome. In this matter, a histopathological investigation of nails could have helped in order to correlate high-frequency sonographic aspects with underlying anatomical changes.
Sandobal et al measured the mean distance between the ventral plate and osseous margin of the distal phalanx and reported also higher values in psoriatic arthritis patients and patients with cutaneous psoriasis compared to rheumatoid arthritis patients [19].

Elongated, twisted, and tortuous capillary loops, of the microvascular dermal plexus, lead to an increased blood flow in both the psoriatic skin and the nail bed [20]. In normal conditions, a minimal quantity of blood flow, due to thin arterial and venous vessels, can be occasionally detected by power Doppler in the nail bed [21]. Power Doppler was also reported as being a feasible highly sensitive tool in monitoring psoriatic plaque treatment response [22].

In our study color and power Doppler imaging revealed increased blood flow in the enlarged vessels in the nail bed of patients with psoriasis compared to controls $(p<0.005)$. This result is in line with those reported by other studies $[13,14]$. When measuring NVRI, significant differences in the NVRI between patients and controls $(p<0.0001)$ and among patients with psoriasis, those with clinical nail involvement had a median NVRI higher than those without nail involvement $(p<0.0001)$. The same NVRI outcome was reported by Husein El-Ahmed et al in a study assessing NVRI in the fourth fingernail of the non-dominant hand in 23 psoriatic patients compared to controls. Interestingly, they stated that they observed a decreased blood supply in the nail bed in psoriatic patients, probably due to dysfunction among endothelial cells and wall vessel thickening [22]. The difference in blood flow supply reports between our study and this study could be explained by the fact that they enrolled patients with psoriasis under systemic treatment with methotrexate or biologic agents at the time of the sonographic evaluation, treatment that could have influenced measurement outcome.

We acknowledge the fact that the ultrasonographic evaluation was performed only by one evaluator and he could not be blinded to the clinical nail findings, but the statistical analysis revealed significant differences despite this limitation. Due to the synovio-entheseal inflammation that occurs in psoriatic arthritis and its anatomic proximity to the nail unit, patients with clinical psoriatic arthritis were not included in this study, in order to assess only nail psoriasis induced changes.

Our findings underline the necessity of correlating clinical modifications with ultrasound aspects in fingernail psoriasis: morphostructural changes in the nail unit could be detected in an early stage, even in clinical nonaffected nails, thus enhancing a precocious diagnosis and a better disease management. Nail bed's blood flow assessment revealed important data regarding microvas- 
culatures alterations and could be taken in consideration as an adequate area to monitor targeted antiangiogenic therapy. This is the first study to report NVRI on a greater number of fingernail psoriasis and we propose this index to be taken into account when an ultrasound evaluation of this area is required.

In conclusion, we found that patients with psoriasis have ventral nail plate deposits, irregular or totally fused nail plates and increased nail plate thickness compared to controls. In patients with psoriasis, an increased blood supply and higher resistance to blood flow were observed in patients with nail involvement compared to those without clinically nail disease. Our observations confirm that a nail unit is a suitable anatomic location for assessing blood flow changes in psoriatic onychopathy using high-frequency sonography with sensitive Power Doppler imaging.

\section{Conflict of interest: None}

Acknowledgement: This paper was published under the frame of the European Social Fund, Human Resources Development Operational Programme 2007-2013, project no. POSDRU/159/1.5/138776

\section{References}

1. Lima XT, Minnillo R, Spencer JM, Kimball AB. Psoriasis prevalence among the 2009 AAD National Melanoma/Skin Cancer Screening Program participants. J Eur Acad Dermatol Venereol 2013; 27: 680-685.

2. Augustin M, Krüger K, Radtke MA, Schwippl I, Reich K. Disease severity, quality of life and health care in plaquetype psoriasis: a multicenter cross-sectional study in Germany. Dermatology 2008; 216: 366-372.

3. Jiaravuthisan MM, Sasseville D, Vender RB, Murphy F, Muhn CY. Psoriasis of the nail: Anatomy, pathology, clinical presentation, and a review of the literature on therapy. $\mathrm{J}$ Am Acad Dermatol 2007; 57: 1-27.

4. Samman PD, Fenton DA. The nails in disease, 5th ed. London: Butterworth-Heinemann Ltd., 1994.

5. Gregoriou S, Kalogeromitros D, Kosionis N, Gkouvi A, Rigopoulos D. Treatment options for nail psoriasis. Expert Rev Dermatol 2008; 3: 339-344.

6. Grover C, Reddy BS, Uma Chaturvedi K. Diagnosis of nail psoriasis: importance of biopsy and histopathology. Br J Dermatol 2005; 153: 1153-1158.

7. Farias DC, Tosti A, Chiacchio ND, Hirata SH. Dermoscopy in nail psoriasis. An Bras Dermatol 2010; 85: 101-103.
8. Micali G, Lacarrubba F, Massimino D, Schwartz RA. Dermatoscopy: Alternative uses in daily clinical practice. J Am Acad Dermatol 2011; 64: 1135-1146.

9. Micali G, Lacarrubba F. Possible applications of videodermatoscopy beyond pigmented lesions. Int J Dermatol 2003; 42: 430-433.

10. Iorizzo M, Dahdah M, Vincenzi C, Tosti A. Videodermoscopy of the hyponychium in nail bed psoriasis. J Am Acad Dermatol 2008; 58: 714-715.

11. Ribeiro CF, Siqueira EB, Holler AP, Fabrício L, Skare TL. Periungual capillaroscopy in psoriasis. An Bras Dermatol 2012; 87: 550-553.

12. Singh R, Bryson D, Singh HP, Jeyapalan K, Dias JJ. Highresolution ultrasonography in assessmentof nail-related disorders. Skeletal Radiol 2012; 41: 1251-1261.

13. Wortsman X, Wortsman J, Carreño L. Sonographic Anatomy of the Skin, Appendages, and Adjacent Structures. In: Wortsman X, Jemec GBE. Dermatologic Ultrasound with Clinical and Histologic Correlations. New York, Springer 2013: 15-38.

14. Gutierrez M, Wortsman X, Filippucci E, De Angelis R, Filosa G, Grassi W. High-frequency sonography in the evaluation of psoriasis: nail and skin involvement. J Ultrasound Med 2009; 28: 1569-1574.

15. Fredriksson T, Pettersson U. Severe psoriasis — oral therapy with a new retinoid. Dermatologica1978; 157: 238-244.

16. Husein El-Ahmed H, Garrido-Pareja F, Ruiz-Carrascosa JC, Naranjo-Sintes R. Vessel resistance to blood flow in the nailfold in patients with psoriasis: a prospective case-control echo Doppler-based study. Br J Dermatol 2012; 166: 54-58.

17. Jäntschi L, Bolboacă SD. Exact probabilities and confidence limits for binomial samples: applied to the difference between two proportions. ScientificWorldJournal 2010; 10: 865-878.

18. Gisondi P, Idolazzi L, Girolomoni G. Ultrasonography reveals nail thickening in patients with chronic plaque psoriasis. Arch Dermatol Res 2012; 304: 727-732.

19. Sandobal C, Carbo E, Iribas J, Roverano S, Paira S. Ultrasound nail imaging on patients with psoriasis and psoriatic arthritis compared with rheumatoid arthritis and control subjects. J Clin Rheumatol 2014; 20: 21-24.

20. Creamer D, Allen MH, Sousa A, Poston R, Barker JN. Localization of endothelial prolife-ration and microvascular expansion in active plaque psoriasis. Br J Dermatol 1997; 136: 859-865.

21. Gutierrez M, Filippucci E, De Angelis R, Filosa G, Kane $\mathrm{D}$, Grassi W. A sonographic spectrum of psoriatic arthritis: "the five targets". Clin Rheumatol 2010; 29: 133-142.

22. Gutierrez M, Filippucci E, Bertolazzi C, Grassi W. Sonographic monitoring of psoriatic plaque. J Rheumatol 2009; 36: $850-851$. 